\title{
Mutations du gène de la polymérase gamma de I'ADN mitochondrial (POLG) associées à l'infertilité masculine?
}

\author{
Isabelle Esther AKNIN-SEIFER 1 2, Renaud-Laurian TOURAINE 2, Jean-Pierre SIFFROI ${ }^{3}$, \\ Clément JIMENEZ ${ }^{4}$, Hervé LEJEUNE ${ }^{5}$, Thierry BIENVENU 6, Catherine PATRAT 7 , \\ Jacques CHOUTEAU ${ }^{8}$, Ken McELREAVEY ${ }^{9}$, Rachel LEVY 1
}

1 Laboratoire de Biologie de la Reproduction, Hôpital Nord et 2 Service de Génétique, Saint-Etienne

3 Service d'Histologie, Biologie de la Reproduction et Cytogénétique (EA1533), Hôpital Tenon, Paris,

4 Laboratoire de Biologie de la Reproduction, CHU Dijon,

5 Département de Médecine de la Reproduction, CHU Hôpital Edouard Herriot, Lyon,

6 Laboratoire de Biochimie et Génétique Moléculaire, Cochin, Paris,

7 Laboratoire Laboratoire de Biologie de la Reproduction, Cochin, Paris,

8 Clinilab, Saint-Martin d'Hères,

9 Reproduction, Fertilité et Populations, Institut Pasteur, Paris.

\section{RESUME}

Le gène POLG, localisé en 15q24-15q26, code pour la sous-unité catalytique de l'ADN polymérase mitochondriale $\gamma$. La partie $\mathbf{N}$ terminale contient une série de 13 glutamines dont les 10 premières sont codées par une répétition du trinucléotide CAG. Cette répétition de CAG est polymorphique, avec un allèle majoritaire de $10 \mathrm{CAG}$ (présent dans $87 \%$ des cas dans la population générale). Rovio et al. ont comparé le nombre de répétitions des triplets CAG dans une population d'hommes " contrôle " (population générale), d'hommes fertiles et différentes populations d'hommes infertiles ; ils ont observé une association entre l'absence de l'allèle normal (correspondant aux 10 répétitions) et l'infertilité masculine, à l'exclusion de l'azoospermie.

Nous avons étudié 503 patients infertiles (dont 84 porteurs d'une microdélétion du chromosome $Y$ ) et 90 témoins fertiles et normospermiques. La région contenant les triplets CAG du gène POLG a été amplifiée et l'analyse de la taille des fragments a été réalisée sur séquenceur.

L'allèle prédominant de 10 répétitions de CAG a été retrouvé chez $\mathbf{9 8 , 9 \%}$ des sujets normospermiques et fertiles de façon homozygote ou hétérozygote (génotype $10 / 10$ ou $10 / x$ ). Parmi les sujets infertiles, nous avons trouvé 14 patients $(3 \%)$ ayant à l'état homozygote un nombre de répétitions différent de 10 : dans 6 cas, les sujets ont deux allèles mutants identiques $(x / x)$, et dans les 8 autres cas, les sujets ont 2 allèles différents (x/y).
Contrairement à l'étude de Rovio et al., parmi les 14 patients homozygotes mutants, deux sont azoospermiques et un est cryptozoospermique. De même, dans notre étude, les sujets infertiles sont aussi souvent hétérozygotes $10 / \times(26,6 \%)$ que les sujets fertiles $(27 \%)$. De plus, un sujet homozygote mutant $12 / 12$ a obtenu une grossesse spontanée ; une seconde grossesse est survenue pendant la prise en charge de son infertilité secondaire. Un autre sujet ayant une mutation a obtenu une grossesse après ICSI, ce qui implique que la mutation n'aurait pas d'effet néfaste sur le développement embryonnaire et l'implantation. A la différence de Rovio et al., un mutant a été trouvé parmi les contrôles fertiles $(1,1 \%)$.

Nos résultats sont les premiers à infirmer ceux de Rovio et al. Ils indiquent qu'il s'agit probablement d'un simple polymorphisme. II est possible que les résultats présentés précédemment par l'équipe de Rovio et al. proviennent d'un biais d'échantillonnage.

Mots clés : infertilité masculine, ADN mitochondrial, polymérase gamma

\section{Correspondance :}

Dr Rachel LEVY - Laboratoire de Biologie de la Reproduction, Hôpital Nord, 42055 Saint-Etienne Tel 04.77.82.83.07 - Email rachel.levy@chu-st-etienne.fr 


\section{INTRODUCTION}

Le gène humain $P O L G$, localisé en 15q24-15q26, code pour la sous-unité catalytique de l'ADN polymérase mitochondriale $\gamma$ (OMIM 174763), qui est la seule polymérase de I'ADN mitochondrial (mt) [3]. La partie $\mathrm{N}$ terminale contient une série de 13 glutamines dont les 10 premières sont codées par une répétition du trinucléotide CAG située dans le premier exon codant du gène. Cette répétition de CAG est polymorphique chez l'homme, avec un allèle majoritaire de 10 CAG ( $87 \%$ dans la population générale) $[4,5]$. II est bien établi que les répétitions CAG sont impliquées dans diverses pathologies humaines, pathologies liées à l'expansion du nombre de répétitions CAG.

Cette répétition de CAG a été étudiée dans des situations de pathologie mitochondriale, délétions multiples ou déplétion de l'ADN mitochondrial $[9,10]$. Seuls quelques cas d'ophtalmoplégie externe progressive (OEP) avec délétions multiples de l'ADN mitochondrial ont été reliés à des mutations faux-sens de POLG (sans modification de la répétition des $\mathrm{CAG}$ ) [6]. L'activité POLG est diminuée in vitro par certaines mutations faux-sens, mais n'est pas modifiée après délétion du motif de polyglutamines [8]. Rovio et al ont comparé le nombre de répétitions des triplets CAG dans une population d'hommes contrôle (population générale), d'hommes fertiles et différentes populations d'hommes infertiles. Leur équipe a observé une association entre l'absence de l'allèle normal (correspondant aux 10 répétitions) et l'infertilité masculine, à l'exclusion de l'azoospermie [7]. Selon cette étude, $9 \%$ des infertilités masculines, hormis les azoospermies et oligozoospermies extrêmes, seraient dues à cette mutation de $P O L G$. Enfin, une étude danoise récente impliquerait le polymorphisme du gène POLG dans l'infertilité inexpliquée avec paramètres spermatiques normaux [2].

\section{MATERIEL ET METHODES}

\section{Patients}

Nous avons étudié 503 patients infertiles (dont 84 porteurs d'une microdélétion du chromosome $Y$ préalablement identifiée) et 90 témoins sains, féconds et normospermiques. Les témoins provenaient de 7 laboratoires en France ; il s'agissait de donneurs de sperme, ou d'homme ayant obtenu une grossesse par insémination intra-conjugale ou fécondation in vitro pour cause strictement féminine (tubaire par exemple), ou encore de volontaires et enfin de candidats à la vasectomie.

Un spermogramme, réalisé pour chaque individu après 3 jours d'abstinence, a été analysé selon les recommandations de l'OMS. Les données cliniques et biologiques (paramètres du spermogramme, bilan hormonal, biochimie du liquide séminal, caryotype) ont permis d'établir la répartition suivante : $27 \%$ d'azoospermies, $36 \%$ d'oligozoospermie extrême $(<1$ million spz $/ \mathrm{ml}), 22 \%$ d'oligozoospermie sévère ( 1 à 5 millions de spz/ml), $6 \%$ d'oligozoospermies modérées ( 5 à 20 millions de $s p z / \mathrm{ml}$ ) et $9 \%$ asthénozoospermie et/ou tératozoospermie avec numération normale.
Dans le cadre de leur bilan d'infertilité, chaque patient a été vu en consultation par un urologue et a bénéficié d'une échographie pelvienne et testiculaire. La répartition des sujets était homogène entre fertiles et infertiles avec une majorité de français d'origine caucasienne et une minorité d'origine nord africaine. L'extraction d'ADN a été réalisée à partir de cellules buccales ou de sang périphérique.

\section{Amplification par PCR}

La région des triplets $C A G$ du gène $P O L G$ a été amplifiée selon les conditions de PCR déjà publiées (amorces mip51 et mip33) avec quelques modifications mineures. L'analyse de la taille des fragments a été réalisée sur un séquenceur CEQ2000 XL (Beckman Coulter France S.A., Roissy, France) et a permis de classer les patients en (i) homozygotes pour l'allèle 10-CAG, (ii) homozygotes mutants en cas d'absence de l'allèle 10-CAG, qu'ils aient deux allèles différents (hétérozygote composite : $x / y$ ) ou un seul allèle (homozygote vrai : $x / x$ ), ou (iii) en hétérozygotes (10/non 10) (Tableau 1).

\section{Analyses statistiques}

Les comparaisons ont été effectuées pour les variables qualitatives par le test du chi2 et le test exact de Fisher quand cela était nécessaire. Une valeur de $p$ inférieure à 0,05 était considérée comme significative.

\section{RESULTATS}

À la différence de Rovio et al, [7] un mutant a été trouvé parmi les contrôles fertiles $(1,1 \%)$. Parallèlement, parmi les 419 patients infertiles idiopathiques (i.e. sans anomalie génétique dépistée et sans étiologie retrouvée), nous avons trouvé 12 " homozygotes mutants " ayant à l'état homozygote un nombre de répétitions différent de 10 (Tableau 2) : dans 6 cas, les sujets ont deux allèles mutants identiques (un 12/12 et cinq 11/11), et dans les 6 autres cas, les sujets ont 2 allèles différents (trois étaient $11 / 12$, deux $9 / 11$ et un 6/9). II est intéressant de noter qu'un sujet homozygote mutant $12 / 12$, oligozoospermique (4 millions de spz/ml) a obtenu une grossesse spontanée ; une seconde grossesse est survenue pendant la prise en charge de son infertilité secondaire. Un autre patient " homozygote mutant " a obtenu une grossesse après ICSI, ce qui impliquerait que la mutation n'aurait pas d'effet néfaste sur le développement embryonnaire et l'implantation. Un autre patient " homozygote mutant " a vu sa numération spermatique se normaliser après chirurgie de sa varicocèle.

Parmi les sujets présentant une microdélétion du chromosome $Y$, nous avons trouvé 2 patients $(2,4 \%)$ ayant à l'état homozygote un nombre de répétitions différent de 10 : dans les 2 cas, les sujets ont 2 allèles différents $(x / y)$.

L'allèle prédominant de 10 répétitions CAG a été retrouvé chez $98,9 \%$ des sujets normospermiques et fertiles de façon homozygote ou hétérozygote (génotype 10/10 ou $10 / x)$. Ainsi, la fréquence des " homozygotes mutants" était de 2,9\% parmi les patients infertiles sans étiologie et 
Tableau 1 : Génotype POLG selon le nombre de répétitions CAG dans les différentes populations masculines.

\begin{tabular}{|c|c|c|c|c|c|c|}
\hline Population & Total & $10 / 10$ & $\begin{array}{l}\text { hétérozygotes } \\
(10 / \text { non10) }\end{array}$ & $\begin{array}{l}\text { "homozygotes } \\
\text { mutants"(a) } \\
\text { (non10/non10) }\end{array}$ & $\begin{array}{c}\text { frequence } \\
\text { attendue des } \\
\text { "homozygotes } \\
\text { mutants" }\end{array}$ & Référence \\
\hline $\begin{array}{l}\text { Hommes fertiles ef } \\
\text { normospermiques }\end{array}$ & $\begin{array}{c}90 \\
(100 \%)\end{array}$ & $\begin{array}{c}65 \\
(72,2 \%)\end{array}$ & $\begin{array}{c}24 \\
(26,7 \%)\end{array}$ & $\begin{array}{c}1 \\
(1,1 \%)\end{array}$ & $2,2 \%$ & présente étude \\
\hline $\begin{array}{l}\text { Hommes infertiles } \\
\text { (tous) }\end{array}$ & $\begin{array}{c}503 \\
(100 \%) \\
\end{array}$ & $\begin{array}{c}352 \\
(70,0 \%) \\
\end{array}$ & $\begin{array}{c}137 \\
(27,2 \%) \\
\end{array}$ & $\begin{array}{c}14 \\
(2,8 \%) \\
\end{array}$ & $2,7 \%$ & présente étude \\
\hline $\begin{array}{c}\text { Hommes infertiles avec } \\
\text { microdélétion du chromosome } Y\end{array}$ & $\begin{array}{c}84 \\
(100 \%)\end{array}$ & $\begin{array}{c}56 \\
(66,7 \%)\end{array}$ & $\begin{array}{c}26 \\
(31,0 \%)\end{array}$ & $\begin{array}{c}2 \\
(2,4 \%)\end{array}$ & $3,4 \%$ & présente étude \\
\hline Hommes fertiles & $\begin{array}{c}98 \\
(100 \%)\end{array}$ & $\begin{array}{c}80 \\
(82 \%)\end{array}$ & $\begin{array}{c}18 \\
(18 \%)\end{array}$ & 0 & $0,9 \%$ & Rovio et al. 2001 [7] \\
\hline $\begin{array}{c}\text { Hommes infertiles: } \\
\text { Totalité }(c) \\
\text { (Finiande, Allemagne, GB) }\end{array}$ & $\begin{array}{c}817 \\
(100 \%)\end{array}$ & $\begin{array}{l}\text { Non } \\
\text { présenté }\end{array}$ & Non présenté & $\begin{array}{c}30 \\
(3,7 \%)\end{array}$ & Non présenté & Rovio et al. 2001 [7] \\
\hline $\begin{array}{l}\text { Hommes non-sélectionnés (d) } \\
\text { (Anglais) }\end{array}$ & $\begin{array}{c}270 \\
(100 \%)\end{array}$ & $\begin{array}{c}196 \\
(73 \%)\end{array}$ & $\begin{array}{c}70 \\
(26 \%)\end{array}$ & $\begin{array}{c}4 \\
(1 \%)\end{array}$ & $2,1 \%$ & Rovio et al. $200\{[7]$ \\
\hline $\begin{array}{l}\text { Hommes non-sélectionnés (d) } \\
\text { (Allemands) }\end{array}$ & $\begin{array}{c}118 \\
(100 \%)\end{array}$ & $\begin{array}{c}90 \\
(76 \%)\end{array}$ & $\begin{array}{c}27 \\
(23 \%)\end{array}$ & $\begin{array}{c}1 \\
(1 \%)\end{array}$ & $1,6 \%$ & Rovio et al. 2001 [7] \\
\hline
\end{tabular}

(a) : La fréquence attendue des "homozygotes mutants" est obtenue selon la loi Hardy-Weinberg à partir de la fréquence du génotype homozygote $10 \mathrm{CAG} / 10 \mathrm{CAG}$;

(b) : Rovio et al. [7] ont sélectionnés une population d'hommes infertiles en excluant les azoospermies et les oligozoospermies extrêmes:

(c) : Nous avons inclus ces 99 hommes sélectionnés dans le total des 817 hommes infertiles de Rovio et al. [7] :

(d) : Les individus non sélectionnés, d'Allemagne et d'Angleterre n'ont pas été caractérisés selon leur fertilité ou leurs paramètres spermatiques.

sans anomalie génétique dépistée (12/419) et plus précisément de $3,5 \%$ et $3,1 \%$ chez les patients avec oligozoospermie et asthénozoospermie.

II est intéressant de souligner que Rovio et al n'ont pas observé d'association entre les mutations de POLG et l'azoospermie ou l'oligozoospermie extrême [7]. Dans notre étude, parmi les 14 patients homozygotes mutants, trois sont azoospermiques, deux sont cryptozoospermiques et deux présentent une oligozoospermie extrême (Tableau 2). Enfin, contrairement à Rovio et al [7], nous avons trouvé une fréquence similaire d'hétérozygotes 10 /non 10 (Tableau 1) chez les sujets infertiles $(26,5 \% ; 111 / 419)$ et chez les sujets féconds $(26,7 \% ; 24 / 90)$.

Aucun des patients " homozygotes mutants " ne présentait de symptomatologie neuromusculaire, en particulier d'ophtalmoplégie.

\section{DISCUSSION}

Nos résultats suggèrent que les répétitions de CAG du gène POLG ne jouent aucun rôle significatif dans l'infertilité masculine. Premièrement, un individu fertile et normospermique a été trouvé " homozygote mutant ". Deuxièmement, un individu "homozygote mutant" présentant une oligozoospermie sévère a obtenu spontanément deux enfants (paternité vérifiée génétiquement). De plus, aucune différence significative n'a été observée (Tableau 1) entre les sujets fertiles et infertiles dans la distribution des trois génotypes ("homozygote mutant", homozygote 10/10 et hétérozygote).

Nous n'avons trouvé que 12 "homozygotes mutants " parmi les 419 patients infertiles sans étiologie reconnue et sans anomalie génétique dépistée, ce qui est en contradiction avec les résultats de Rovio et al [7] dans lesquels 9 "homozygotes mutants" avaient été identifiés sur 99 hommes infertiles. Nous estimons que le nombre supérieur de patients inclus dans notre étude permet d'éviter un biais de recrutement. Toutefois, des études complémentaires restent nécessaires pour éliminer des polymorphismes éventuels en étudiant des individus d'origines géographiques diverses.

Comme nous avons identifié comme " homozygotes mutants " 7 patients avec azoospermie, cryptozoospermie ou oligozoospermie extrême, il nous semble licite d'inclure dans les calculs de fréquence toutes les populations d'homme infertiles, quels que soient leurs paramètres spermatiques. Ainsi, si l'on totalise les 817 patients infertiles de l'étude de Rovio et al provenant de Finlande, Allemagne, Grande Bretagne et Irlande [7], la fréquence d' " homozygotes mutants " pour leur étude tombe de $9 \%$ à $3,7 \%$, ce qui est comparable à nos résultats (Tableau 1 ).

Dans notre étude, la fréquence observée $(2,9 \%)$ des " homozygotes mutants " parmi les hommes infertiles, est proche de la fréquence attendue $(2,5 \%)$, calculée suivant la loi de Hardy-Weinberg, à partir de la fréquence des homozygotes 10-10. Dans notre population d'hommes fer- 
Tableau 2 : Phénotype et génotype des 12 hommes infertiles * homozygotes mutants ».

\begin{tabular}{|c|c|c|c|c|c|c|c|c|c|c|c|c|}
\hline Patients & MES-A & BLA-B & Y87-C & MEL-D & MEK-E & ZAH-F & HAD-G & BER-H & BAS-1 & PB-J & MAC-K & MOU-L \\
\hline Age & 39 & 29 & 32 & 36 & 38 & 39 & 36 & 28 & 35 & 32 & 35 & 35 \\
\hline $\begin{array}{l}\text { Origine } \\
\text { ethnique (a) }\end{array}$ & $F$ & $\mathrm{~F}$ & $F$ & $\mathrm{~F}$ & AK & $M$ & A & $F$ & $F$ & $F$ & $F$ & A \\
\hline Numération (b) & $<1000$ & $3 \times 106$ & $<1000$ & $0,012 \times 106$ & $4 \times 106$ & 0,24106 & 0 & 0 & 0 & 7,106 & $11 \times 106$ & $4 \times 106$ \\
\hline Mobilité $(\%)(c)$ & $\therefore$ & 30 & $\therefore$ & 25 & 60 & 40 & $\therefore$ & - & - & 20 & 15 & 30 \\
\hline $\begin{array}{c}\text { Formes } \\
\text { anormales }(\%)\end{array}$ & - & 61 & - & - & 70 & 83 & - & - & - & 94 & 86 & 40 \\
\hline FSH Ul/ic (d) & ND & 4,1 & 45,9 & 3,9 & 5 & 8,9 & 16,7 & 15,1 & 21.5 & 15 & 7,7 & 10,4 \\
\hline LH UIIId (e) & ND & ND & ND & 2,9 & 6,7 & 6 & ND & 4,3 & 7,9 & 4,7 & 2,7 & 4,7 \\
\hline $\begin{array}{l}\text { Testostérone } \\
\text { ng/mle (f) }\end{array}$ & ND & 5,89 & $\$ 1,2$ & 17 & 7,91 & 2,9 & ND & $\begin{array}{c}200 \mathrm{ng} / \mathrm{dl} \\
\text { (ibre) }\end{array}$ & 5,4 & 4 & 3,49 & 3.69 \\
\hline Inhibine B ng/l (g) & ND & 103 & ND & 95 & 85 & 15 & ND & 38 & $<15$ & ND & ND & ND \\
\hline Caryotype & $46 X Y$ & $46 X Y$ & $46 X Y$ & $46 X Y$ & $46 X Y$ & $46 X Y$ & $46 X Y$ & $46 \times Y$ & $46 X Y$ & $46 X Y$ & $46 X Y$ & $46 X Y$ \\
\hline Grossesse & Non & Non & $\begin{array}{l}\text { Insémination avec } \\
\text { sperme de donneur }\end{array}$ & $\begin{array}{c}\text { Grossesse en } \\
\text { cours après } \\
\text { ICSI }\end{array}$ & Non & Non & $?$ & Non & Non & $\begin{array}{l}1 \text { enfant sain } \\
\text { par ICSI }\end{array}$ & $\begin{array}{c}2 \mathrm{ICSI}, \\
\text { pas de } \\
\text { grossesse }\end{array}$ & $\begin{array}{c}2 \text { grossesses } \\
\text { spontanees }\end{array}$ \\
\hline Mutation POLG & $6-9$ & $9-11$ & $9-11$ & $11-12$ & $11-12$ & $11-12$ & $11-11$ & $11-11$ & $11-11$ & $11-11$ & $11-11$ & $12-12$ \\
\hline
\end{tabular}

(a) : Origine : $F=$ Français $: A K=$ Algérien-Kabyle $: M=$ Marocain $; A=$ Algérien :

(b) : Nombre de spermatozoïdes par $\mathrm{ml}$ :

(c) : \% de mobilité $(a+b)$ selon les recommandations de l'OMS (rapides progressifs + progressifs lents); normale > 50\%;

(d) : FSH normale de 2 à $10 \mathrm{Ul} / \mathrm{l}$ :

(e) : LH normale de 2 à $10,9 \mathrm{UI} / \mathrm{l}$;

(f) : Testostérone normale de 2,43 à $8,32 \mathrm{ng} / \mathrm{ml}$ :

(g) : Inhibine B normale $>100 \mathrm{ng} / \mathrm{l}$;

ND : Non disponible.

tiles, la fréquence observée d' " homozygotes mutants " $(1,1 \%)$ est inférieure à celle attendue $(2,2 \%)$, mais cette différence est non significative, probablement due à un effectif limité de la population contrôle fertile. Cette remarque est également valable pour la population des 98 hommes fertiles de l'étude de Rovio et al, où aucun " homozygote mutant " n'avait été observé alors que un était attendu [7]. Deux études récentes conduites en Italie ont donné des résultats similaires aux nôtres, ne montrant aucun rôle significatif du polymorphisme de $P O L G$ dans l'infertilité masculine [4 ; Guarducci et al : résultats présentés au Symposium International European Academy of Andrology "Genetics of Male Infertility : from Research to Clinic", Florence, Italie, 2003].

Enfin, si les hommes " homozygotes mutants " avaient effectivement une fertilité réduite, une réduction progressive de ce génotype à travers les générations serait attendue et en l'absence d'un quelconque avantage sélectif pour les individus hétérozygotes, le génotype « homozygote mutant " devrait être extrêmement rare de nos jours.

De plus, nos résultats sont en accord avec l'absence d'effet délétère visible sur la réplication du génome mitochondrial de la suppression expérimentale de la répétition CAG [8]. D'ailleurs, le motif glutamine est absent dans les protéines polg du rat et de la souris, alors qu'une répétition plus courte est retrouvée chez le gorille avec 6 CAG, et chez le chimpanzé avec 4 et 7 CAG (Genebank access numbers NM017462, NM053528, AF415155, AF415156, AF415157) [1], ce qui est fréquemment observé pour d'autres gènes.
Enfin, lorsque les répétitions CAG sont impliquées dans diverses pathologies humaines, celles-ci sont dues à une expansion de la répétition CAG.

En conclusion, notre étude ne confirme aucune relation entre le polymorphisme des répétitions $C A G$ dans le gène POLG et l'infertilité masculine.

\section{CONCLUSION}

Nos résultats sont les premiers à infirmer ceux de Rovio et al [7]. Ils indiquent qu'il s'agit probablement d'un simple polymorphisme. II est possible que les résultats présentés précédemment par l'équipe de Rovio et al [7] proviennent d'un biais d'échantillonnage.

\section{Remerciements :}

Nous remercions le comité organisateur de la SALF pour le prix attribué à ce travail présenté sous forme de poster lors du congrès de la SALF, à Orléans.

\section{REFERENCES}

1. HANCOCK J.M., WORTHEY E.A., SAINTIBANEZ-KOREF M.F. : A role for selection in regulating the evoluionary emergence of disease-causing and other coding CAG repeats in humans and mice. Mol. Biol. Evel., 2001, 18: 1014-1023. 
2 JENSEN M., LEFFERS H., PETERSEN J.H. et al. : Frequent polymorphism of the mitochondrial DNA polymerase gamma gene (POLG) in patients with normal spermiograms and unexplained subfertility. Hum. Reprod., 2004, $19: 65-70$.

3. LECRENIER N., FOURY F. : New features of mitochondrial DNA replication system in yeast and man. Gene 2000, 246 : $37-48$.

4. MICHIELOTTO C., STUPPIA L., FORESTA G. et al. : The CAG-repeat polymorphism of the POLG1 gene is not associated with male oligozoospermia. Am. J. Hum. Gen., 2003, 2435.

5. ROPP P.A., COPELAND W.C. : Cloning and characterization of the human mitochondrial DNA polymerase, DNA polymerase gamma. Genomics, 1996, $36: 449-458$.

6. ROVIO A., TIRANTI V., BEDNARZ A.L. et al. : Analysis of the trinucleotide CAG repeat from the human mitochondrial DNA polymerase gene in healthy and diseased individuals. Eur. J. Hum. Genet., 1999, 7 : 140-146.

7. ROVIO A.T., MARCHINGTON D.R., DONAT S. et al. : Mutations at the mitochondrial DNA polymerase (POLG) locus associated with male infertility. Nat. Genet., 2001, 29 : 261262.

8. SPELBRINK J.N., TOIVONEN J.M., HAKKAART G.A et al. : In vivo functional analysis of the human mitochondrial DNA polymerase POLG expressed in cultured human cells. J. Biol. Chem., 2000, $275: 24818-24828$.

9. VAN GOETHEM G., DERMAUT B., LOFGREN A. et al. Mutation of $P O L G$ is associated with progressive external ophthalmoplegia characterized by mtDNA deletions. Nat. Genet., 2001, $28: 211-212$.

10. VAN GOETHEM G., MARTIN J.J., VAN BROECKHOVEN C. Progressive external ophthalmoplegia characterized by multiple deletions of mitochondrial DNA : unraveling the pathogenesis of human mitochondrial DNA instability and the initiation of a genetic classification. Neuromol. Med., 2003, 3 : 129-146.

Poster primé au $X X^{\circ}$ Congrès de la Société d'Andrologie de Langue Française, Orléans, $11-13$ décembre 2003.

Manuscrit reçu : avril 2004 ; accepté juin 2004.

\section{ABSTRACT}

The CAG repeat of mitochondrial DNA polymerase gamma (POLG) is not associated with male infertility. A French multicentre study

Isabelle Esther AKNIN-SEIFER, Renaud-Laurian TOURAINE, Jean-Pierre SIFFROI, Clément JIMENEZ, Hervé LEJEUNE, Thierry BIENVENU, Catherine PATRAT, Jacques CHOUTEAU, Ken McELREAVEY, Rachel LEVY

The single DNA polymerase gene of mitochondrial DNA (POLG) contains a polymorphic CAG repeat in the first coding exon. The promising results of a first study by Rovio et al. showing that several cases of male infertility were related to a "homozygous mutant" genotype with a number of CAG repeats differing from 10, prompted us to conduct a large study on 503 infertile and $\mathbf{9 0}$ fertile men.

Our results do not confirm any significant relationship between the polymorphic CAG repeat in the POLG gene and male infertility.

Key-Words : polymerase gamma, mitochondrial DNA, male infertility 